\section{Modes of Escape from Therapeutic Control in Myelomatosis}

\section{J. R. HOBBS}

In the first myeloma trial of the M.R.C. working party on leukaemia in adults the deaths that have occurred can be attributed to unrelated diseases $(5 \%$-for example, coronary thrombosis, etc.); causes indirecily lelated to the tumour ( $8 \%$-for example, pneumonia due to the immune paresis); no obvious response of the tumour to treatment $(27 \%)$; and finally relapse of the tumour after an initial response to treatment $(60 \%)$.

In analysing this last group it became clear that in nearly half of these patients the relapsing tumour was more aggressive than it had been initially. This occurred more often in those patients whose paraprotein levels fell rapidly (to under half the initial level within three months) than in those where a slower response to cytotoxic drugs was observed. The rapid responders also showed initially lower levels of serum albumin, perhaps reflecting their faster initial growth rates and protein production. A fast response could be due to a loss of the normal resting phase (memory cells) of antibody-forming cells, and where this is apparently retained in the slow responders a better prognosis is assured.

During the relapse four types of "escape" could be distinguished. Firstly, $45^{\circ} \%$ of the $60 \%$ showed a change to a faster growth rate (growth rate escape). This group included the other types of escape-secondly, 35\% showing the appearance de novo or an increased proportion of Bence Jones production (Bence Jones escape); thirdly, 5\%, a clear change in the paraprotein pattern (mutation escape); and, fourthly, 3\%, loss of paraprotein production reverting to a "reticulosarcoma" (non-paraprotein escape).

These changes (like the induction of monocytic leukaemia) mostly require 18 months or longer to appear, a period compatible with a mutagenic action of the cytotoxic drugs. Longcontinued cytotoxic treatment should therefore be reserved only for patients (especially so in childhood) who would otherwise die of their disease.

Department of Chemical Pathology, Westminster Hospital, London S.W.1

J. R. HOBBS

\section{Total Body Irradiation for Myelo- matosis}

\section{E. BERGSAGEL}

Treatment with melphalan and cyclophosphamide has improved the outlook for patients with plasma cell neoplasms. These drugs cause the tumour to regress in 30 to $50 \%$ of patients, and prolong survival significantly. As I began to look at these data more critically it became clear that the improved survival could be explained by a tumour cell kill of less than $1 \mathrm{log}$.

The average tumour cell kill achieved by melphalan therapy may be estimated in several ways. Firstly, by dividing the prolongation of survival attributed to this drug by an estimate of the average tumour doubling time. On the assumption that the rate of increase in the $M$-protein concentration in the serum and urine reflects the growth rate of the tumour, and from the studies of Hobbs and Salmon-which suggest that the $M$ protein doubling time of most $\mathrm{G}$ and $\mathrm{A}$ myelomas is about six months-the results of these calculations suggest that the improved survival attributed to melphalan could be explained by the inhibition of 1.83 tumour doublings.

The second method of evaluating the tumour cell kill is in the individual patient by following the regrowth of the tumour after effective treatment has been discontinued. We have discontinued giving melphalan to three patients in whom the $M$ protein had disappeared completely from the serum and the urine and then determined the interval required for this to increase to its pretreatment level; the $M$-protein doubling time was then calculated. We found that therapy which inhibits the growth of less than 1 log. of tumour cells can prolong survival significantly because these tumours regrow so slowly. When I showed these data to a radiotherapist he pointed out that total body irradiation should be equally effective, for the tolerated single dose of 225 to 300 rads should reduce the number of tumour cells by about $1 \mathrm{log}$.

The in vivo sensitivity of a mouse plasma cell tumour, using a cell culture method to assay the surviving fraction of myeloma stem cells capable of forming colonies, revealed a $\mathrm{D}_{n}(37 \%$ survival) of 110 rads. The maximum decrease in tumour mass observed in a patient following the irradiation of several skin plasmacytomas with varying doses also indicated a $D_{0}$ of about 110 rads. Hence the radiation sensitivity of the mouse and human myeloma tumour cells appears to be very similar to other dividing mammalian cells.

\section{Haematological Toxicity}

The haematological toxicity resulting from total body irradiation is the chief factor limiting the safe dose of irradiation that can be used. With marrow transplantation in identical twins it has been possible to irradiate the recipient with as much as 1,500 rads. Patients with plasma cell myeloma would be significantly benefited if the dose of total body irradiation could be raised to 1,000 rads. With this dose a tumour cell kill of 3 logs would be expected, and if the tumour doubling time during regrowth was three months a remission of 30 months could be expected. With a regrowth doubling time of 6 months the remission would last for 60 months, and if the doubling time of were as long as 12 months a remission of 10 years would be expected.

Nevertheless, if doses of 1,000 rads of total body irradiation are to be used some method of protecting the patient's marrow function must be worked out. We are considering two methods. Firstly, to give the total body irradiation in two doses, protecting an appreciable amount of the marrow, such as the pelvis, during the first irradiation. Probably haemopoietic progenitor cells circulate in the blood, and these cells could be expected to repopulate irradiated areas. The protected area of the pelvis could then be irradiated during a second stage, after normal marrow function had recovered in the irradiated areas.

The second approach would be to obtain a large sample of marrow from the patient before irradiation, and reinfuse the haemopoietic precursor cells, separated from the larger, denser myeloma cells, after irradiation. My colleagues at The Ontario Cancer Institute have been able to separate myeloma cells from haemopoietic precursor cells by density gradient centrifugation in Ficoll, a uncharged polysaccharide of high molecular weight and also by velocity gradient sedimentation in the Staput apparatus.

Princess Margaret Hospital, Toronto, Ontario, Canada D. E. BERGSAGEL 